\title{
Incidence and risk factors of suicide reattempts within I year after psychiatric hospital discharge in mood disorder patients
}

This article was published in the following Dove Press journal:

Clinical Epidemiology

21 November 201।

Number of times this article has been viewed

\author{
Chidchanok Ruengorn' \\ Kittipong Sanichwankul ${ }^{2}$ \\ Wirat Niwatananun ${ }^{3}$ \\ Suwat Mahatnirunkul ${ }^{2}$ \\ Wanida Pumpaisalchai ${ }^{2}$ \\ Jayanton Patumanond' \\ 'Clinical Epidemiology Unit, Faculty \\ of Medicine, Chiang Mai University; \\ ${ }^{2}$ Suanprung Psychiatric Hospital; \\ ${ }^{3}$ Department of Pharmaceutical Care, \\ Faculty of Pharmacy, Chiang Mai \\ University, Chiang Mai, Thailand
}

Background: The incidence and risk factors of suicide reattempts within 1 year after psychiatric hospital discharge in mood disorder patients remain uninvestigated in Thailand.

Objective: To determine incidence and risk factors of suicide reattempts within 1 year after psychiatric hospital discharge in mood disorder patients.

Methods: A retrospective cohort study was conducted by reviewing medical charts at Suanprung Psychiatric Hospital, Chiang Mai, Thailand. Mood disorder patients, diagnosed with the International Statistical Classification of Diseases and Related Health Problems 10th Revision codes F31.x, F32.x, and F33.x, who were admitted owing to suicide attempts between October 2006 and May 2009 were eligible. The influence of sociodemographic and clinical risk factors on suicide reattempts was investigated using Cox's proportional-hazards regression analysis.

Results: Of 235 eligible mood disorder patients, 36 (15.3\%) reattempted suicide (median 109.5 days, range 1-322), seven (3.0\%) completed suicide (median 90 days, range 5-185), and $192(84.2 \%)$ neither reattempted nor completed suicide during follow-up. Of all nonfatal suicide reattempts, 14 patients $(38.9 \%)$ did so within 90 days. Among suicide completers, one $(14.3 \%)$ did so 5 days after discharge, and four (57.1\%) did so within 90 days. The following three risk factors explained $73.3 \%$ of the probability of suicide reattempts: over two previous suicide attempts before the index admission (adjusted hazard ratio [HR] 2.48; 95\% confidence interval [CI] 1.07-5.76), being concomitantly prescribed typical and atypical antipsychotics (adjusted HR 4.79; 95\% CI 1.39-16.52) and antidepressants, and taking a selective serotonin reuptake inhibitor alone (adjusted HR 5.08; 95\% CI 1.14-22.75) or concomitantly with norepinephrine and/or serotonin reuptake inhibitors (adjusted HR 6.18; 95\% CI 1.13-33.65).

Conclusion: Approximately $40 \%$ of suicide reattempts in mood disorder patients occurred within 90 days after psychiatric hospital discharge. For mood disorders and when there have been over two previous suicide attempts, prescribed antipsychotics or antidepressants may help predict suicide reattempts.

Keywords: bipolar disorders, major depressive disorders, suicide attempts, suicide completions

\section{Introduction}

Mood disorders are disturbances in a person's mood categorized into major depressive disorder (MDD) and bipolar disorder (BD). MDD and BD appear to be the most important risk factors for lifetime suicide in any psychiatric disorder. ${ }^{1}$ It has been estimated that at the time of suicide, $87.3 \%-98 \%$ of psychiatric disorders are from mood disorders and substance abuse. ${ }^{2}$

Among mood disorder patients who attempt suicide, reattempt is more common than the first act. ${ }^{3}$ The prevalence of reattempted suicide within 1 year after the index
Correspondence: Jayanton Patumanond Clinical Epidemiology Unit, Faculty of Medicine, Chiang Mai University, Chiang Mai 50200, Thailand

Tel +6653946306

Fax +66 53945476

Email j.patumanond@yahoo.com 
suicide attempt is $16 \%-40 \%$ nonfatal and $2 \%$ fatal. ${ }^{4,5}$ Risk of reattempted suicide following a psychiatric admission is highest during the first months after the attempt and declines over time. $^{6}$

Several risk factors for suicide attempts reported in MDD and BD patients include male sex, ${ }^{7,8}$ age, ${ }^{5,7}$ living alone on the day of the index attempt, ${ }^{9}$ smoking, ${ }^{10}$ alcohol abuse, ${ }^{9}$ previous number of suicide attempts, ${ }^{9-12}$ method of suicide attempt, ${ }^{9}$ severity of refractory or recurrent depressions, ${ }^{10,12}$ somatic illness, ${ }^{9}$ suicide intent and treatment of mental illness, ${ }^{7,9,11}$ hospitalization in a psychiatric department, and the use of psychopharmacological drugs during the follow-up period. Other risk factors are impulsivity, stressful life events, hopelessness, being unemployed, ${ }^{13}$ comorbidity with anxiety and agitation, and reporting suicidal plans or hallucinations at the time of the index episode. ${ }^{14}$

The current study was undertaken at a setting located in the upper northern part of Thailand where the suicide attempt rate of 34.1 per 100,000 population is remarkably high. The suicide completion rate was the highest in the nation at 12.4 per 100,000 population compared with 5.97 per 100,000 population countrywide in 2009. Mood disorders rank among the top five high-burden diseases in this setting every year. Among those diagnosed with mood disorders, $25 \%$ are hospitalized due to suicide attempts. ${ }^{15}$ Suicide reattempts are prevalent in this vulnerable population; however, the incidence and risk factors of suicide reattempts in mood disorder patients have not yet been investigated in this area. Furthermore, consensus on suicide attempt risk factors is still unclear because of their complexity, and the generalizability of previous research may be limited due to discrepancies of cultural context, study populations, design, and data analysis. Thus, there is a need for studies of incidence and risk factors for suicide reattempts in mood disorder patients in Thailand. The present study aimed to determine incidence and explored risk factors of suicide reattempts occurring within 1 year after psychiatric hospital discharge in mood disorder patients.

\section{Methods}

\section{Study population and setting}

Suanprung Psychiatric Hospital is the largest tertiary psychiatric hospital ( 700 beds) in the upper northern region of Thailand. It is located in Chiang Mai and has been providing medical care since 1938. In 2009, the hospital provided services to 6721 inpatients and 61,561 outpatients. Mood disorder inpatient and outpatient visits for 2007, 2008, and 2009 were $9069,10,739$, and 11,482 , respectively. ${ }^{16}$
Eligible patients were all mood disorder patients diagnosed by psychiatrists and coded by the International Statistical Classification of Diseases and Related Health Problems 10th Revision (ICD-10). Inclusion criteria were patients diagnosed with ICD-10 codes F31.x, F32.x, and F33.x and admitted to Suanprung Psychiatric Hospital owing to suicide attempts between October 2006 and May 2009.

\section{Outcome definitions and measures}

People who reattempted suicide were those mood disorder patients who reattempted nonfatal suicide within 1 year after an admission to Suanprung Psychiatric Hospital owing to attempted suicide. Eligible patients' medical charts were examined for the main outcome of the study, which was time to suicide reattempts. Time to suicide reattempts was defined as the number of days within 1 year from the date of hospital discharge to the suicide reattempt date. Patients who did not reattempt suicide during the 1 year of follow-up or with whom contact was lost before the 1-year follow-up were considered a censor group. Their time at risk from the index date to censoring time was collected. Further, the national death registration was searched and death date and cause of death were retrieved. A cause of death was further confirmed by telephone calls to the patient's spouse or next of kin. To determine risk factors for suicide reattempts, suicide completers were excluded from the analysis, because this population did not overlap.

\section{Data collection}

The study was designed as a retrospective cohort study. Patients' medical files were reviewed from the day of admission and followed for 1 year after hospital discharge. The admission time was set as an index date. Patient information recorded in hospital files was routinely assessed and documented with a standardized procedure by psychiatrists, trained psychiatric nurses, and pharmacists who interviewed patients and/or their relatives. Five trained nurses working at the study site extracted data and filled in a case record form. To assure validity of the data, two meetings were held by the research team to discuss and clarify each variable in the files before data collection. Undocumented variables were considered to be missing.

Sociodemographic factors and clinical factors were the main indicators collected in the study. All factors were selected and divided into categories based on a literature review and the opinion of psychiatrists on the research team. The sociodemographic factors included were sex, age, body mass index, marital status, educational level, occupation, 
religion, living status (alone or with others), number of children, and summation of number of stressful life events right before or at the index episode. Social support was divided into good or excellent, moderate, and little or very little.

Clinical factors included ICD-10 diagnoses of BD (coded F31.x) and MDD (coded F32.x and F33.x). Duration of treatment began from the time of the first diagnosis to the index date, and age at onset was the age at the first visit. Previous admission referred only to admission to Suanprung Psychiatric Hospital before the index date. Previous suicide attempt and previous suicidal ideation were assessed and counted from the first hospital visit to the index date. Psychotic symptoms at an index episode included hallucination and hearing voices. Psychotic comorbidity was extracted from the ICD-10 code and was recorded and grouped into alcohol/substance dependence, which was commonly found, and others. Somatic illness was any chronic disease. Smoking, alcohol use, and any substance abuse were behaviors at the index date. Medication adherence was assessed by attending pharmacists asking patients or their relatives about consistency in taking medication during the 2 weeks before the index date. It was summarized as "highly adhere," meaning the patient always took medication or rarely missed a dose, "intermittently adhere," meaning the patient took medication off and on, and "poorly adhere," meaning at least seven consecutive days when the patient did not take medication. Other clinical characteristics included family history of mental disorders and family history for attempted or completed suicide.

An additional variable collected was the number and type of method(s) used for suicide attempt at the index date. The method was considered a violent method if it was (1) a method other than drug overdose or a single wrist cut or (2) a combination of different methods. ${ }^{17}$ Treatments received during the admission included pharmacotherapy, electroconvulsive therapy, and psychotherapy. Pharmacotherapy was prescribed medication, which we categorized according to the mechanism of action and evidence of relation to suicide. Mood stabilizers were categorized either as lithium or others, such as carbamazepine and valproate. Antidepressants were grouped as norepinephine and/or serotonin reuptake inhibitors, such as amitriptyline and venlafaxine, or as selective serotonin reuptake inhibitors (SSRIs), such as fluoxitine and fluvoxamine. Antipsychotics were divided into typical, such as zuclopentizol and haloperidol, and atypical, such as clozapine and risperidone. Anxiolytics consisted of benzodiazepine and others, such as chlodiazepoxide. Psychotherapy included individual and group counseling, family counseling, and rehabilitation. Additionally, data on length of stays and readmission within 28 days were also collected.

\section{Statistical analyses}

Because time to suicide reattempts was an outcome of the study, Cox's proportional-hazards regression was used for the analysis. Owing to the nature of the fitting exploration model, we determined the best parsimonious model by performing two steps of analysis. First, variables predicting suicide reattempts were explored using univariable Cox's proportional-hazards regression. Those with a $P$-value less than 0.20 were selected for the last step, in which forward elimination multivariable Cox's proportional-hazards regression was selected. The global goodness-of-fit test by Schoenfeld was used for testing the proportional-hazards assumption. ${ }^{18}$ In order to assess the model prediction, we used the area under receiver operating characteristic curve method. The statistical significance level was set at 0.05 . Two institutional review boards, the Faculty of Medicine at Chiang Mai University and Suanprung Psychiatric Hospital, approved the protocol of the study.

\section{Results}

A total of 235 patients' medical folders were reviewed and included in the analysis (Figure 1). Of those, 36 (15.32\%) reattempted suicide (median 109.5 days, range 1-322), seven (2.98\%) completed suicide (median 90 days, range 5-185), and $192(84.21 \%)$ neither reattempted nor completed suicide during follow-up. Of all nonfatal suicide reattempts, 14 patients (38.9\%) did so within 90 days. Among suicide completers, one $(14.3 \%)$ did so 5 days after discharge, and four $(57.1 \%)$ did so within 90 days. Total time of follow-up in each group is presented in Table 1.

Univariable analysis found that stressful life events, psychotic symptoms, and previous suicide attempts were significantly associated with suicide reattempts (Tables 2 and 3).

Three risk factors that explained $73.3 \%$ of the probability of suicide reattempt were attempting suicide more than twice before the index admission (adjusted hazard ratio [HR] 2.48; 95\% confidence interval [CI] 1.07-5.76], concomitantly prescribed typical and atypical antipsychotics (adjusted HR 4.79; 95\% CI 1.39-16.52), or taking SSRI alone (adjusted HR 5.08; 95\% CI 1.14-22.75) or with norepinephrine and/or serotonin reuptake inhibitors (adjusted HR 6.18; 95\% CI 1.13-33.65). In addition, readmission within 28 days was significantly higher in the suicide reattempt group (18.8\% vs $5.1 \%, P=0.008$ ) (data not shown) (Table 4). 


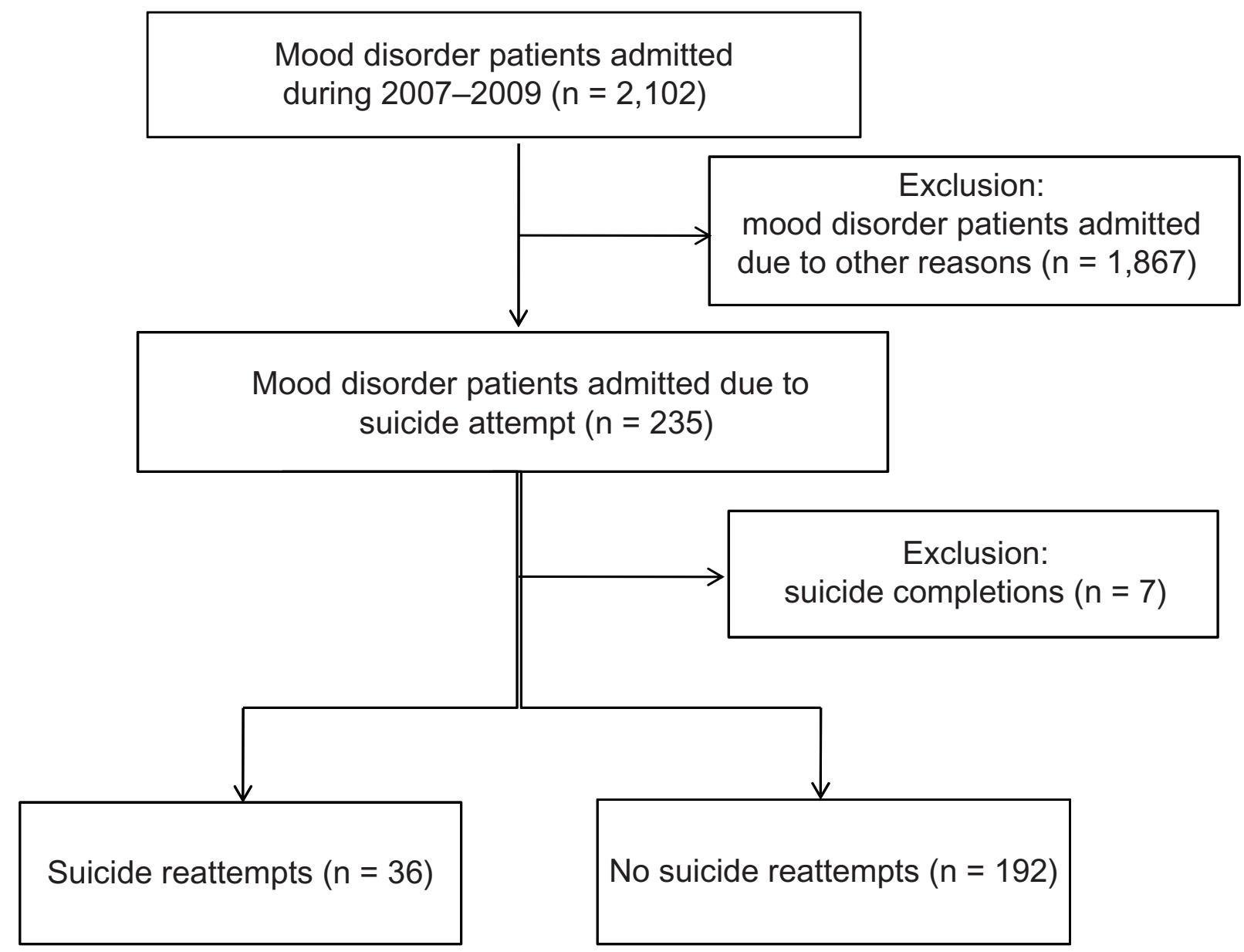

Figure I Data flow.

\section{Discussion}

To the best of our knowledge, the present study is the first to determine incidence and explore risk factors of suicide reattempts within 1 year after psychiatric hospital discharge in mood disorder patients. In this study, the incidence of suicide reattempts and suicide completions within 1 year of follow-up after attempted suicide was $15.3 \%$ and $3.0 \%$, respectively. This finding is consistent with previous studies that reported suicide reattempts at $13.6 \%-16 \%{ }^{6,13}$ and suicide completions at $1 \%-3 \% .{ }^{19}$ Although the median time of suicide reattempts in the present study was longer than in a previous study (109.5 days vs 73.5 days), ${ }^{13}$ almost $40 \%$ of nonfatal suicide reattempts of the patients in this study occurred within 90 days. The reattempt rate is more likely to be clustered in time because the incidence declines throughout the year of follow-up. ${ }^{6}$ Therefore, intensive surveillance, timely assessment, and effective interventions should be emphasized during the first few months and continue for 1 year after hospital discharge.

Using the best fit and simple model, we found three risk factors that indicated suicide reattempts: more than two previous suicide attempts before the index admission, concomitantly being prescribed typical and atypical antipsychotics, and taking an SSRI alone or concomitantly with norepinephrine and/or serotonin reuptake inhibitors. Previous suicide attempts before the index date is a risk

Table I Incidence of suicide reattempts and suicide completions within I year after hospital discharge in mood disorder patients $(n=235)$

\begin{tabular}{llll}
\hline Parameters & Suicide reattempts $(\mathbf{n}=\mathbf{3 6})$ & Suicide completions $(\mathbf{n}=\mathbf{7})$ & No reattempts $(\mathbf{n}=1 \mathbf{9 2})$ \\
\hline Cumulative incidence $(\%)$ & 15.32 & 2.98 & \\
Incidence rate per 1000 population & 8.45 & 9.65 & 35,616 \\
Total person-days at risk & 4261 & 725 & $168.5(1-365)$ \\
Median days (min-max) & $109.5(I-322)$ & $90(5-185)$ & \\
\hline
\end{tabular}


Table 2 Sociodemographic factors of mood disorder patients and unadjusted hazard ratio (HR) (95\% confidence interval [Cl]) of suicide reattempts by univariable Cox's proportional-hazards regression analysis $(n=228)$

\begin{tabular}{|c|c|c|c|c|}
\hline \multirow[t]{2}{*}{ Sociodemographic risk factors ${ }^{a}$} & \multicolumn{2}{|c|}{ Suicide reattempts } & \multirow{2}{*}{$\begin{array}{l}\text { Unadjusted HR } \\
(95 \% \mathrm{Cl})\end{array}$} & \multirow[t]{2}{*}{$P$-value } \\
\hline & Yes $(n=36)$ & No $(n=192)$ & & \\
\hline Sex & & & & 0.882 \\
\hline Male & $16(17.8)$ & $74(82.2)$ & 1.0 & \\
\hline Female & $20(14.5)$ & $118(85.5)$ & $0.95(0.49-1.84)$ & \\
\hline Age (years) & $36.8( \pm \mid 3.9)$ & $37.7( \pm \mid 4.4)$ & $0.99(0.97-1.01)$ & 0.388 \\
\hline Body mass index $\left(\mathrm{kg} / \mathrm{m}^{2}\right)$ & $21.8( \pm 3.3)$ & $22.2( \pm 4.1)$ & $0.97(0.89-1.06)$ & 0.553 \\
\hline \multicolumn{5}{|l|}{ Education } \\
\hline No or primary school & $17(17.0)$ & $83(83.0)$ & 1.0 & \\
\hline Junior to high school & $14(20.0)$ & $56(80.0)$ & $1.24(0.6 \mathrm{I}-2.52)$ & 0.550 \\
\hline Diploma or higher & $5(11.1)$ & $40(88.9)$ & $0.62(0.23-1.68)$ & 0.346 \\
\hline \multicolumn{5}{|l|}{ Marital status } \\
\hline Single & $12(15.4)$ & $66(84.6)$ & 1.0 & \\
\hline Married & $16(14.3)$ & $96(85.7)$ & $0.79(0.37-1.67)$ & 0.536 \\
\hline Widow/divorce or others & $8(22.2)$ & $28(77.8)$ & $1.34(0.55-3.29)$ & 0.516 \\
\hline \multicolumn{5}{|l|}{ Occupation } \\
\hline Officer & $18(19.1)$ & $76(80.9)$ & 1.0 & \\
\hline Labor & $8(16.3)$ & $4 \mid(83.7)$ & $0.95(0.4 \mathrm{I}-2.20)$ & 0.913 \\
\hline Housewife/student & $2(7.1)$ & $26(92.9)$ & $0.31(0.07-1.34)$ & 0.116 \\
\hline Unemployed & $8(14.8)$ & $46(85.2)$ & $0.86(0.37-1.97)$ & 0.713 \\
\hline \multicolumn{5}{|l|}{ Religion } \\
\hline Buddhism & $35(16.6)$ & $176(83.4)$ & 1.0 & \\
\hline Others & I (I2.5) & $7(87.5)$ & $0.74(0.10-5.4 \mathrm{I})$ & 0.769 \\
\hline \multicolumn{5}{|l|}{ Number of children } \\
\hline None & $7(15.6)$ & $38(84.4)$ & 1.0 & \\
\hline One to two & $4(7.4)$ & $50(92.6)$ & $0.54(0.16-1.86)$ & 0.330 \\
\hline More than two & $4(21.0)$ & $15(79.0)$ & $1.32(0.39-4.52)$ & 0.656 \\
\hline \multicolumn{5}{|l|}{ Living status } \\
\hline Alone & $2(11.8)$ & $15(88.2)$ & 1.0 & \\
\hline With family & $31(17.7)$ & $144(82.3)$ & $1.23(0.30-5.17)$ & 0.773 \\
\hline With others & I (5.6) & $17(94.4)$ & $0.48(0.04-5.29)$ & 0.549 \\
\hline \multicolumn{5}{|l|}{ Social support } \\
\hline Good or excellent & $19(17.3)$ & 91 (82.7) & 1.0 & \\
\hline Moderate & $2(6.7)$ & $28(93.3)$ & $0.43(0.10-1.86)$ & 0.262 \\
\hline Very little or little & $12(20.0)$ & $48(80.0)$ & $1.18(0.57-2.43)$ & 0.654 \\
\hline \multicolumn{5}{|l|}{ Stressful life events } \\
\hline None & $10(33.3)$ & $20(66.7)$ & 1.00 & \\
\hline One event & $14(13.6)$ & $89(86.4)$ & $0.38(0.17-0.87)$ & 0.021 \\
\hline Two events & $5(8.5)$ & $54(91.5)$ & $0.23(0.08-0.68)$ & 0.008 \\
\hline More than two events & $7(19.4)$ & $29(80.6)$ & $0.53(0.20-1.39)$ & 0.196 \\
\hline
\end{tabular}

Note: ${ }^{a}$ Numbers are $\mathrm{n}(\%)$ or mean $( \pm$ standard deviation).

factor associated with suicide reattempts found in several studies. ${ }^{9-12}$ In the present study, $74.4 \%$ percent of patients had at least one prior suicide attempt before the index date. The hazard ratios increased with an increasing number of previous suicide attempt(s), demonstrating that patients with more prior attempts tended to be more severe, be more impulsive, and take more risks. ${ }^{20}$

Regarding the use of medication, psychopharmacological use prior to and during the follow-up period is a predictor for suicide attempt. ${ }^{6}$ Suicide reattempts increased in mood disorder patients prescribed with antipsychotics or antidepressants. This is in accordance with a meta-analysis study reporting that antidepressants were not beneficial for suicidal patients. ${ }^{21}$ However, in some previous studies, ${ }^{22}$ long-term treatment with antidepressants was found to reduce suicides significantly when combined with lithium and antipsychotics. Antipsychotic users were more likely to reattempt suicide in this study. The ability of most antipsychotics to limit the risk of suicidal behavior remains untested in the literature. ${ }^{23}$ The positive relationship results of antidepressant and antipsychotic use in this study may be due to confounding indications. Patients prescribed with more types of medication were those who were more severe and prone to a higher number of suicide reattempts. 
Table 3 Clinical factors of mood disorder patients and unadjusted hazard ratio (HR) ( $95 \%$ confidence interval [Cl]) of suicide reattempts by univariable Cox's proportional-hazards regression analysis $(n=228)$

\begin{tabular}{|c|c|c|c|c|}
\hline \multirow[t]{2}{*}{ Clinical factors ${ }^{a}$} & \multicolumn{2}{|c|}{ Suicide reattempts } & \multirow{2}{*}{$\begin{array}{l}\text { Unadjusted HR } \\
(95 \% \mathrm{Cl})\end{array}$} & \multirow[t]{2}{*}{$P$-value } \\
\hline & Yes $(n=36)$ & No $(n=192)$ & & \\
\hline \multicolumn{5}{|l|}{ ICD-I0 } \\
\hline Bipolar & $9(17.0)$ & $44(83.0)$ & 1.0 & \\
\hline Major depressive disorder & $27(15.4)$ & I $48(84.6)$ & $1.00(0.47-2.12)$ & 0.993 \\
\hline \multicolumn{5}{|l|}{ Somatic illness } \\
\hline No & $26(16.2)$ & I 34 (83.8) & 1.0 & \\
\hline One disease & $7(13.2)$ & $46(86.8)$ & $0.90(0.39-2.08)$ & 0.812 \\
\hline Two or more diseases & $3(20.0)$ & $12(80.0)$ & $1.39(0.42-4.49)$ & 0.592 \\
\hline \multicolumn{5}{|l|}{ Psychotic comorbidity } \\
\hline No & $28(16.3)$ & I 44 (83.7) & 1.0 & \\
\hline Yes & $6(18.2)$ & $27(81.8)$ & $1.24(0.5 \mathrm{I}-3.00)$ & 0.631 \\
\hline \multicolumn{5}{|l|}{ Age under 18 years at onset } \\
\hline No & $2(10.5)$ & $17(89.5)$ & 1.0 & \\
\hline Yes & $33(17.7)$ & $153(82.3)$ & I.4I (0.34-5.90) & 0.634 \\
\hline \multicolumn{5}{|l|}{ Year(s) of treatment } \\
\hline$\leq 5$ & $24(14.1)$ & $146(85.9)$ & 1.0 & \\
\hline$>5$ & II (29.0) & $27(71.0)$ & $1.95(0.95-3.98)$ & 0.067 \\
\hline \multicolumn{5}{|l|}{ Previous admission } \\
\hline No & $17(1 \mathrm{l} .4)$ & I $32(88.6)$ & 1.0 & \\
\hline Yes & $18(23.4)$ & $59(76.6)$ & $1.94(1.00-3.77)$ & 0.051 \\
\hline \multicolumn{5}{|l|}{ Psychotic symptoms ${ }^{b}$} \\
\hline No & $27(18.2)$ & I2I (8I.8) & 1.00 & \\
\hline Yes & $5(7.8)$ & $59(92.2)$ & $0.36(0.14-0.94)$ & 0.037 \\
\hline \multicolumn{5}{|l|}{ Mental disorders in family } \\
\hline No & $21(13.4)$ & $136(86.6)$ & 1.0 & \\
\hline First-degree relatives & $6(35.3)$ & II (64.7) & $2.39(0.96-5.92)$ & 0.060 \\
\hline Other relatives & $8(19.0)$ & $34(81.0)$ & $1.40(0.62-3.17)$ & 0.415 \\
\hline \multicolumn{5}{|l|}{ Suicide in family } \\
\hline No & $31(15.1)$ & $174(84.9)$ & 1.0 & \\
\hline Yes & $4(25.0)$ & $12(75.0)$ & $2.03(0.7 \mathrm{I}-5.77)$ & 0.184 \\
\hline \multicolumn{5}{|l|}{ Previous suicide attempts } \\
\hline None & $10(12.2)$ & $72(87.8)$ & 1.0 & \\
\hline One to two times & II (I2.0) & $81(88.0)$ & $0.95(0.40-2.24)$ & 0.912 \\
\hline More than two times & I5 (28.3) & $38(71.7)$ & $2.31(1.04-5.19)$ & 0.039 \\
\hline \multicolumn{5}{|l|}{ Previous suicidal ideation } \\
\hline None & $9(13.0)$ & $60(87.0)$ & 1.0 & \\
\hline One to two times & II (II.6) & $84(88.4)$ & $1.03(0.43-2.49)$ & 0.946 \\
\hline More than two times & $16(26.2)$ & $45(73.8)$ & $2.23(0.98-5.06)$ & 0.055 \\
\hline \multicolumn{5}{|l|}{ Lethality prior to admission } \\
\hline No & $21(17.6)$ & $98(82.4)$ & 1.0 & \\
\hline Yes & $15(13.9)$ & $93(86.1)$ & $0.73(0.38-1.43)$ & 0.364 \\
\hline \multicolumn{5}{|l|}{ Smoking } \\
\hline No & $26(15.4)$ & $143(84.6)$ & 1.0 & \\
\hline Yes & $10(24.4)$ & $31(75.6)$ & $1.53(0.74-3.17)$ & 0.255 \\
\hline \multicolumn{5}{|l|}{ Alcohol drinking } \\
\hline No & $22(15.5)$ & $120(84.5)$ & 1.0 & \\
\hline Yes & $14(20.6)$ & $54(79.4)$ & $1.55(0.79-3.03)$ & 0.200 \\
\hline Any substance abuse & & & & \\
\hline No & $33(17.0)$ & $161(83.0)$ & 1.0 & \\
\hline Yes & $3(18.8)$ & I3 (8I.2) & I.II (0.34-3.62) & 0.864 \\
\hline Medication adherence & & & & \\
\hline Highly & $25(15.9)$ & $132(84.1)$ & 1.0 & \\
\hline Intermittent & $8(34.8)$ & $15(65.2)$ & I.82 (0.82-4.05) & 0.138 \\
\hline Poor & I (5.0) & $19(95.0)$ & $0.38(0.050-2.78)$ & 0.338 \\
\hline Antidepressant & & & & \\
\hline No & $2(4.9)$ & $39(95.1)$ & 1.0 & \\
\hline
\end{tabular}


Table 3 (Continued)

\begin{tabular}{|c|c|c|c|c|}
\hline \multirow[t]{2}{*}{ Clinical factors ${ }^{a}$} & \multicolumn{2}{|c|}{ Suicide reattempts } & \multirow{2}{*}{$\begin{array}{l}\text { Unadjusted HR } \\
(95 \% \mathrm{Cl})\end{array}$} & \multirow[t]{2}{*}{$P$-value } \\
\hline & Yes $(n=36)$ & No $(n=192)$ & & \\
\hline Norepinephrine/serotonin & $2(10.0)$ & $18(90.0)$ & $2.40(0.34-17.06)$ & 0.381 \\
\hline Serotonin alone & $26(18.3)$ & $116(81.7)$ & $3.46(0.82-14.60)$ & 0.090 \\
\hline Both & $6(24.0)$ & $19(76.0)$ & $4.13(0.83-20.49)$ & 0.082 \\
\hline \multicolumn{5}{|l|}{ Antipsychotic } \\
\hline No & $5(8.5)$ & 54 (9।.5) & 1.0 & \\
\hline Typical & $22(17.5)$ & $104(82.5)$ & $2.08(0.79-5.5 \mathrm{I})$ & 0.139 \\
\hline Atypical & $3(17.6)$ & $14(82.4)$ & $1.68(0.40-7.05)$ & 0.475 \\
\hline Both & $6(23.1)$ & $20(76.9)$ & $2.63(0.80-8.66)$ & 0.110 \\
\hline \multicolumn{5}{|l|}{ Anxiolytics } \\
\hline No & $10(19.6)$ & $4 \mathrm{I}(80.4)$ & 1.0 & \\
\hline Benzodiazepine & $22(16.1)$ & $115(83.9)$ & $1.09(0.5 \mathrm{I}-2.30)$ & 0.829 \\
\hline Others & $2(11.1)$ & $16(88.9)$ & $0.78(0.17-3.54)$ & 0.744 \\
\hline Both & $2(9.1)$ & $20(90.9)$ & $0.57(0.12-2.60)$ & 0.468 \\
\hline \multicolumn{5}{|l|}{ Mood stabilizer } \\
\hline No & $25(16.2)$ & $129(83.8)$ & 1.0 & \\
\hline Lithium & $6(15.8)$ & $32(84.2)$ & $0.84(0.34-2.05)$ & 0.705 \\
\hline Others & $5(13.9)$ & $31(86.1)$ & $0.80(0.31-2.10)$ & 0.659 \\
\hline \multicolumn{5}{|l|}{ Electroconvulsive therapy } \\
\hline No & $25(15.1)$ & 140 (84.9) & 1.0 & \\
\hline Yes & II (18.0) & $50(82.0)$ & $1.06(0.52-2.17)$ & $0.86 I$ \\
\hline \multicolumn{5}{|l|}{ Hospital stay (days) } \\
\hline Median (min-max) & $14.5(2-50)$ & $13(1-135)$ & $1.00(0.98-1.02)$ & 0.992 \\
\hline
\end{tabular}

Notes: aNumbers are $\mathrm{n}(\%)$ or mean ( \pm standard deviation); bat onset.

Abbreviation: ICD- I0, International Statistical Classification of Diseases and Related Health Problems I0th Revision.

Stressful life events and psychotic symptoms were two risk factors associated with suicide reattempts found in the univariable analysis but not in the multivariable analysis. This is possibly because of the intercorrelation with one or more other variables. A previous study indicated that stressful life events play an important role in suicide attempts in patients with mood disorders, particularly

Table 4 Risk factors of suicide reattempts and adjusted hazard ratio $(\mathrm{HR})(95 \%$ confidence interval $[\mathrm{Cl}])$ by multivariable Cox's proportional-hazards regression analysis $(n=228)$

\begin{tabular}{lll}
\hline Risk factors & Adjusted HR (95\% Cl) & P-value \\
\hline Previous suicide attempts & & \\
$\quad$ None & 1.00 & \\
$\quad$ One to two times & $0.95(0.40-2.27)$ & 0.909 \\
$\quad$ More than two times & $2.48(1.07-5.76)$ & 0.034 \\
Antipsychotic & & \\
$\quad$ No & 1.00 & 0.120 \\
$\quad$ Typical & $2.18(0.82-5.79)$ & 0.265 \\
Atypical & $2.30(0.53-9.95)$ & 0.013 \\
Both & $4.79(1.39-16.52)$ & \\
Antidepressant & & \\
$\quad$ No & 1.00 & 0.167 \\
$\quad$ Norepinephrine/serotonin & $4.11(0.55-30.60)$ & 0.030 \\
Selective serotonin & $5.08(1.14-22.75)$ & \\
reuptake inhibitor & & 0.035 \\
Both & $6.18(1.13-33.65)$ & \\
\hline
\end{tabular}

MDD patients. ${ }^{24}$ We found that having psychotic symptoms at the time of admission reduced the probability of suicide attempts, which is consistent with a previous study that suggested that psychosis may impair planning for suicide attempts. ${ }^{25}$

Currently, there are a few studies that determine risk factors of suicide reattempts in mood disorders. Most of the research reports risk factors associated with suicide attempts in MDD or BD separately. Therefore, inconsistencies between our findings and previous studies are partially due to the difference in population, methodology, assessment tools, statistical analysis, and small samples of some variables in this study. Other studies have found risk factors for suicide attempts that include male sex; ${ }^{7,8}$ increasing age (ie, 50 years or older) ${ }^{5,7}$ living alone on the day of the index attempt; ${ }^{9}$ the method used in the index attempt; ${ }^{9,26}$ prior admission for suicide reattempt; ${ }^{8,9,19}$ family history of mental disorders; ${ }^{27}$ previous suicidal ideation; ${ }^{25}$ somatic illness, such as cardiovascular disease and malignancies; ${ }^{7,28}$ abuse of psychopharmacological agents, alcohol, or substances; ${ }^{28,29}$ bipolar, $^{30,31}$ depressed, and mixed episodes among BD patients; recurrent or refractory MDD, including those with psychosis; ${ }^{32}$ a positive family history of suicidal behaviors ${ }^{27}$ not taking lithium; and short-term effects from electroconvulsive therapy. ${ }^{33}$ 
There are some limitations of the present study. First, the generalizability of the study results may be limited to mood disorder patients visiting psychiatric hospitals. Those with severe physical injuries resulting from the attempted suicide were likely to be referred to a general hospital. Their characteristics, such as lethality of the method used and severity of psychiatric illness, may be different. Second, data collected from medical folders were subject to incompleteness or missing variables, which caused a lack of power to identify associations. Third, the data relied exclusively on patient interview. Some information, such as drug and alcohol use, can be under-reported. Additionally, the effect of some variables, such as psychotic disease in family members or a family history of suicide, could be underestimated. These variables may be missed because patients were unaware of them, or they may remain unspoken due to stigma. Some factors were not assessed in this study by instruments used in other studies (eg, hopelessness and impulsiveness). Thus, their potential risk was not directly detected. Fourth, we used variables at the admission index date as risk factors. However, some factors can change over time and occur during follow-up, such as marital status, employment situation, development of depression, and medication adherence. These may have affected our findings. However, the aim of the study was to examine factors at one specific point in time that predict a high risk of repeated suicide attempts and thus possibly guide treatment. Finally, we found a final model that moderately explained the probability of suicide reattempts $(73.3 \%)$. A larger sample size is required in future research to increase statistical power and should be prospectively used to confirm the study results.

In spite of some limitations, risk factors of suicide reattempts in mood disorder patients were identified. For future research, risk factors for long-term suicide reattempts must be investigated, because they may differ somewhat from the short-term risk factors found in the present study. Furthermore, risk factors categorized by subgroup of mood disorders (ie, MDD and BD) must be separately investigated to enable clinicians to provide intervention specifically directed for each subgroup. Finally, this study lacks statistical power to investigate risk factors for suicide completion and suicidal ideation. Future research on those issues is warranted.

\section{Conclusion}

Almost $40 \%$ of suicide reattempts in mood disorder patients in this study occurred within 90 days after their discharge from a psychiatric hospital. Factors placing patients at high risk for suicide reattempts were over two previous suicide attempts before the index admission, concomitantly being prescribed typical and atypical antipsychotics and antidepressants, and taking an SSRI alone or concomitantly with norepinephrine and/or serotonin reuptake inhibitors.

\section{Acknowledgment}

This study was financially supported in part by the Faculty of Medicine and the Graduate School, Chiang Mai University.

\section{Disclosure}

All authors have no conflicts of interest in this research.

\section{References}

1. Simon RI, Hales RE. Suicide Risk: Assessing the Unpredictable. 1st ed. Simon RI, Hales RE, editors. Arlington, VA: American Psychiatric Publishing, Inc; 2006.

2. Harris CE, Barraclough BM. Suicide as an outcome for mental disorders. Br J Psychiatry. 1997;170:205-228.

3. Kerkhof A. Attempted Suicide: Patterns and Trends. 1st ed. Hawton K, van Heeringen K, editors. New York: John Wiley \& Sons, Ltd; 2000.

4. Hawton K, Fagg J. Suicide, and other causes of death, following attempted suicide. Br J Psychiatry. 1988;152:359-366.

5. Nordström P, Samuelsson M, Åsberg M. Survival analysis of suicide risk after attempted suicide. Acta Psychiatr Scand. 1995a;91:336-340.

6. Christiansen E, Jensen BF. Risk of repetition of suicide attempt, suicide or all deaths after an episode of attempted suicide: a register-based survival analysis. Aust N Z J Psychiatry. 2007;41:257-265.

7. Soukas J, Suominen K, Isometsä E, et al. Long-term risk factors for suicide mortality after attempted suicide: findings of a 14-year follow-up study. Acta Psychiatr Scand. 2001;104:117-121.

8. Skogman K, Alsén M, Öjehagen A. Sex differences in risk factors for suicide after attempted suicide: a follow-up study of 1052 suicide attempters. Soc Psychiatry Psychiatr Epidemiol. 2004;39:113-120.

9. Cooper J, Kapur N, Webb R, et al. Suicide after deliberate self-harm: a 4-year cohort study. Am J Psychiatry. 2005;162:297-303.

10. Oquendo MA, Galfalvy H, Russo S, et al. Prospective study of clinical risk factors of suicidal acts after a major depressive episode in patients with major depressive disorder or bipolar disorder. Am J Psychiatry. 2004;161:1433-1441.

11. Owens D, Wood C, Greenwood DC, et al. Mortality and suicide after non-fatal self-poisoning: 16-year outcome study. $\mathrm{Br} J$ Psychiatry. 2005; 187:470-475.

12. Oquendo MA, Currier D, Mann JJ. Prospective studies of suicidal behavior in major depressive and bipolar disorders: what is the evidence for predictive risk factors? Acta Psychiatr Scand. 2006;114(3):151-158.

13. Kapur N, Cooper J, King-Hele S, et al. The repetition of suicidal behavior: a multicenter cohort study. J Clin Psychiatry. 2006; 67(10):1599-1609.

14. Schneider B, Philipp M, Muller MJ. Psychopathological risk factors of suicide in patients with major depression during a 5-year follow-up. Eur Psychiatry. 2001;16(5):283-288.

15. Suanprung Psychiatric Hospital. Suanprung's outpatient and inpatient services treat most psychiatric illnesses. Chiang Mai, Thailand; 2010 [updated 2010]. Available from: http://www.suanprung.go.th/eng/ top_five.php. Accessed November 7, 2011.

16. Suanprung Psychiatric Hospital. Suanprung psychiatric hospital report number of patients between 2007 to 2009. Chiang Mai, Thailand; 2010 [updated 2010]. Available from: http://www.suanprung.go.th/statt/ index.html. Accessed November 7, 2011. 
17. Träskman L, Åsberg M, Bertilsson L, Sjöstrand L. Monoamine metabolites in CSF and suicidal behaviour. Arch Gen Psychiatry. 1981;38:631-636.

18. Greenland S. Introduction to Regression Modeling. Modern Epidemiology. 3rd ed. Rothman KJ, et al, editors. Philadelphia: Lippincott Williams \& Wilkins; 2008:418-455.

19. Sakinofsky I. Treating suicidality in depressive illness. Part I: current controversies. Can J Psychiatry. 2007;52(6 Suppl 1):71S-84S.

20. Corruble E, Damy C, Guelfi JD. Impulsivity: a relevant dimension in depression regarding suicide attempts. J Affect Disorders. 1999;53:211-215.

21. Stone M, Laughren T, Jones ML, et al. Risk of suicidality in clinical trials of antidepressants in adults: analysis of proprietary data submitted to US Food and Drug Administration. BMJ. 2009;339:b2880.

22. Angst F, Stassen HH, Clayton PJ, Angst J. Mortality of patients with mood disorders: follow-up over 34-38 years. $J$ Affect Disord. 2002;68(2-3):167-181.

23. Baldessarini RJ, Pompili M, Tondo L. Suicide in bipolar disorder: risks and management. CNS Spectr. 2006;11(6):465-471.

24. Suppapitiporn $\mathrm{S}$. The risk factors of suicidal attempt in depressed patients. Chulalongkorn University; 2002 [updated 2002; cited November 26, 2009]; Thesis (MSc) - Chulalongkorn University, 2002]. Available from: http://cuir.car.chula.ac.th/dspace/handle/123456789/6093. Accessed November 7, 2011.
25. Oquendo MA. Suicidal behavior in bipolar mood disorder: clinical characterisic of attempters and nonattempetrs. J Affect Disord. 2000;59:107-117.

26. Suominen K, Isometsä E, Suokas J, et al. Completed suicide after a suicide attempt: a 37-year follow-up study. Am J Psychiatry. 2004;161:563-564.

27. Lizardi D, Sher L, Sullivan GM, et al. Association between familial suicidal behavior and frequency of attempts among depressed suicide attempters. Acta Psychiatr Scand. 2009;119(5):406-410.

28. Nielsen B, Wang AG, Bille-Brahe U. Attempted suicide in Denmark. IV. A five-year follow-up. Acta Psychiatr Scand. 1990; 81:250-254.

29. Murphy GE, Wetzel RD. The lifetime risk of suicide in alcoholism. Arch Gen Psychiatry. 1990;47:383-392.

30. Bottlender R, Jaeger M, Strauss A, Möller HJ. Suicidality in bipolar compared to unipolar depressed inpatients. Eur Arch Psychiatry Clin Neurosci. 2000;250(5):257-261.

31. Fiedorowicz JG. Do risk factors for suicide behavior differ by affective disorder polarity? Psychol Med. 2009;39(5):763-771.

32. Scoliers G, Portzky G, van Heeringen K, Audenaert K. Sociodemographic and psychopathological risk factors for repetition of attempted suicide: a 5-year follow-up study. Arch Suicide Res. 2009; 13(3):201-213.

33. Sharma V. Retrospective controlled study of inpatient ECT: does it prevent suicide? J Affect Disord. 1999;56(2-3):183-187.
Clinical Epidemiology

\section{Publish your work in this journal}

Clinical Epidemiology is an international, peer-reviewed, open access journal focusing on disease and drug epidemiology, identification of risk factors and screening procedures to develop optimal preventative initiatives and programs. Specific topics include: diagnosis, prognosis, treatment, screening, prevention, risk factor modification, systematic

\section{Dovepress}

reviews, risk \& safety of medical interventions, epidemiology \& biostatical methods, evaluation of guidelines, translational medicine, health policies \& economic evaluations. The manuscript management system is completely online and includes a very quick and fair peer-review system, which is all easy to use. 